\title{
STRESS ANALYSIS FOR THE MANDIBULAR SCREW-RETAINED FULL ARCH ACRYLIC PROSTHESIS WITH DIFFERENT CANTILEVER EXTENSION (3D FINITE ELEMENT ANALYSIS)
}

\author{
Moaz Mostafa Ahmed Farrag* , Doaa Amr Rostom** and Hamdy Abo Elfotouh Hamed***
}

\begin{abstract}
Background: Implant-retained restorations offer numerous advantages over regular removable dentures. Basically, efficient retention and stability is easily achieved by constructing a fixed restoration or at least by using overdenture attachments instead of depending on the weak physical means used with regular dentures.

Methods: A geometrical model demonstrating screw retained acrylic fixed restoration, mucosa, implants, abutment, screw, alveolar bones were generated to accomplish the assessments. The parameters presented in the computer model were cantilever extent and stresses with 50 and $100 \mathrm{~N}$ performed to mimic the occlusal stresses. geometrics with the peripheries of stress were gained and the extreme stress at each site was designed in diagrams for analysis.
\end{abstract}

Results: Stresses clustered at the elements closest to the loading point. implant deformation shows a reduction with the increase of cantilever length.

Conclusion: Alveolar bone received less stresses and deformation under more rigid restoration that it distributes the applied load in a good manner than lower rigidity one.

KEY WORDS: Implant, Stress, Cantilever, Finite, Mandible.

\section{INTRODUCTION}

Implant-retained restorations offer numerous advantages over regular removable dentures. Essentially, higher retention-stability complex was simply accomplished by fabricating a fixed restoration or minimally by overdenture attachments as an alternative of reliant on the fragile physical means used with regular dentures ${ }^{1}$. Such improvement had an implication on improving masticatory efficiency. In reviews, it was ${ }^{2}$ determined that the grouping of a lower implant overdenture

\footnotetext{
* Dental Specialist, Ministry of Health.

** Lecturer of Prosthodontics, Faculty of Dentistry, Cairo University.

*** Professor of Prosthodontics, Faculty of Dentistry, Cairo University
} 
and upper conventional complete denture provides objectable increase in the masticatory performance in relation to complete dentures in both arches. The improved retention allows also for reduction in the restoration size which is a great benefit for new denture wearers, gaggers and patients with tori. Minimizing soft tissue coverage allows also for improved taste sensation. All the previous factors contribute to increase patient gratification and improve quality of life when treated with implant retained restorations ${ }^{3-4}$.

Theoretically, distal cantilevers exert bending moment on the bio-system affecting the prosthesis, implants and the surrounding bone. Bending overload is accused for biological complications manifested mainly with bone loss at the most distal implant. Bending moments has been also blamed for many prosthetic complications as screw loosening, screw fracture, prosthesis fracture and in some cases fracture of the implant body ${ }^{5,6}$.

Off-axial application of forces as in cantilevered implant supported prostheses- induces bending moments within the entire load-bearing system according to the cantilever arm extension. The longer the distance, the greater was the bending moment ${ }^{7}$. Jacques et $\mathrm{al}^{20}$ investigated the role of cantilever extent on the force spreading of lower-cantilevered implant-supported restoration using a strain gauge method. Frameworks were fabricated and attached into ordinary abutments located on a definitive-cast containing five implant duplications. Two linear strain gauges were attached on the mesial and distal surfaces of each abutment to detect deformation. A vertical static stress of 100 Newton was released to the cantilever arm at the distances of 10,15 , and 20 $\mathrm{mm}$ from the middle of the distal abutment and the entire values of exact deformation were documented. They found that the abutment deformation was higher with longer cantilever extensions specially with $20 \mathrm{~mm}$ cantilever which drove the conclusion that excessively long cantilever should be avoided.
Rubo and Souza ${ }^{8}$ conducted a similar study by using three dimensional finite element analysis. A prototype revealing the anterior segment of a lower arch restored with 5 fixtures reinforcing a structure was generated to achieve the finiteanalysis. The computer was preset with the physical characteristics of the materials as described in the literature, and 100 Newton vertical stress was used to mimic the occlusal forces. Loads at the fixtureabutment interface were increased by $45 \%$ when the cantilever extent was higher from 10 to $15 \mathrm{~mm}$ and by $30 \%$ from 15 to $20 \mathrm{~mm}$, mainly double times the stress revealed with a $10 \mathrm{~mm}$ cantilever.

3D finite element analysis was performed to mimic actual strains that may enhance bone alteration in two specific models. Effects in preserving bone bulk under implant-retained overdentures based on this laboratory research, the addition of posterior short implants has a satisfactory effect in preserving bone bulk under implant-retained overdentures.

The aim of the study is to accomplish a comparative study between different cantilever lengths regarding the stress distribution in mandibular implant- supported acrylic restoration by using finite element analysis.

\section{METHODS}

This stress analysis study using finite element method performed at faculty of dentistry, Cairo University to compare between different screwretained fixed restorations on 4 dental implants with different cantilever length $(10,16,20) \mathrm{mm}$ to obtain the main outcome which is stress distribution in implant-bone interface in mega-pascal (MPA) using finite element software.

The finite element model components as the screw retained fixed restoration, mucosa, implants, abutment, screw, cortical and cancellous bones were created in "Autodesk Inventor" edition 8 (Autodesk Inc., San Rafael, CA, USA), then exported as STEP files. These components were assembled in ANSYS 
environment (ANSYS Inc., Canonsburg, PA, USA). The system analyzed in this investigation consisted of the commonly available root form threaded titanium dental implant (Bio-Horizons, USA) with straight multi-unit abutments. The root form dental implant had a nominal diameter of $4.2 \mathrm{~mm}$ (Implant Tapered 4.2 with internal hex, Platform 4.5), length of $15 \mathrm{~mm}$ and $12 \mathrm{~mm}$.

That implants were located at their planned positions at the lateral incisors and pre-molar regions. The two anterior implants (with $15 \mathrm{~mm}$ length) were perpendicular to the horizontal plane of the superior border of the mandible while maintaining the inter-implant parallelism. The two posterior implants (with $12 \mathrm{~mm}$ length) were also placed vertically, while perfect osseointegration, was assumed to be presented between implants and bone.

The simulated peri-implant bone included an inner layer representing cancellous bone of 22 $\mathrm{mm}$ height and $14 \mathrm{~mm}$ width covered by an outer thin layer of cortical bone of $2 \mathrm{~mm}$ thickness. The mucosa thickness was $2 \mathrm{~mm}$. The screw retained fixed restoration was simulated of height $8 \mathrm{~mm}$ and width of $8.8 \mathrm{~mm}$, where three materials were tested as; porcelain fused to metal (PFM), and prosthetic acrylic.

Implant complex, mandible and their assembly as appeared on Inventor screen. All these parts in addition to the implant, abutment, clips and bar were exported from Inventor as STEP files. Then set of Boolean operations were carried out to assemble all the model components before meshing.

Group of designing processes between the components were extablished before obtaining the final model(s) designed. The meshing of these components was done by $3 \mathrm{D}$ solid element (SOLID187) which has three degrees of freedom (translation in main axes directions).

The resultant stresses of both vertical and oblique loading conditions were collected and tabulated according to maximum values of Von Mesis stress (Stress equivalent) which indicate the resultant stresses in Mega Pascal (MPa) and total deformation in millimeter $(\mathrm{mm})$ on each component in a specified volume (implant, attachment, mucosa, cortical bone, ... etc.). In this study, the ANSYS program presented the maximal resultant Von Mesis stress values in red color, while the minimal values were represented in blue color, as showed in figure (1), (2), (3) and (4).

The obtained results were demonstrated and compared. In each run (case study) each model component results were taken as screen shots from ANSYS. The definition of most important results obtained and demonstrated below as follows;

$\mathrm{S}_{1}$ : Maximum principal (Max tensile) stress

$\mathrm{S}_{3}$ : Minimum principal (Max Compressive) stress

$\mathrm{S}_{\text {shear }}:$ Max shear Stress

$\mathrm{S}_{\text {von }}:$ Von Mises (Equivalent) stress

$\mathrm{U}_{\text {sum }}$ : Total deformation

$\mathrm{U}_{\mathrm{z}}$ Deformation in $\mathrm{Z}$ direction (vertical)

In the following sections, the obtained results and their comparisons was demonstrated;

Acrylic screw-retained fixed restorations, Bilateral vertical loading of $100 \mathrm{~N}$ at first molars, $50 \mathrm{~N}$ at second premolars, $10 \mathrm{~mm}$ cantilever length.

In this case, an implant mandibular fixed restoration was tested as the restoration was supported by these implants two at lateral incisors and two at the first pre-molar. The vertically applied load was $100 \mathrm{~N}$ at first molars and $50 \mathrm{~N}$ second premolars bilaterally, where cantilever length is $10 \mathrm{~mm}$ while the fixed restoration material was "Acrylic resin". The following figures are just listing of the different deformation and stresses distributions for each component of the model. 


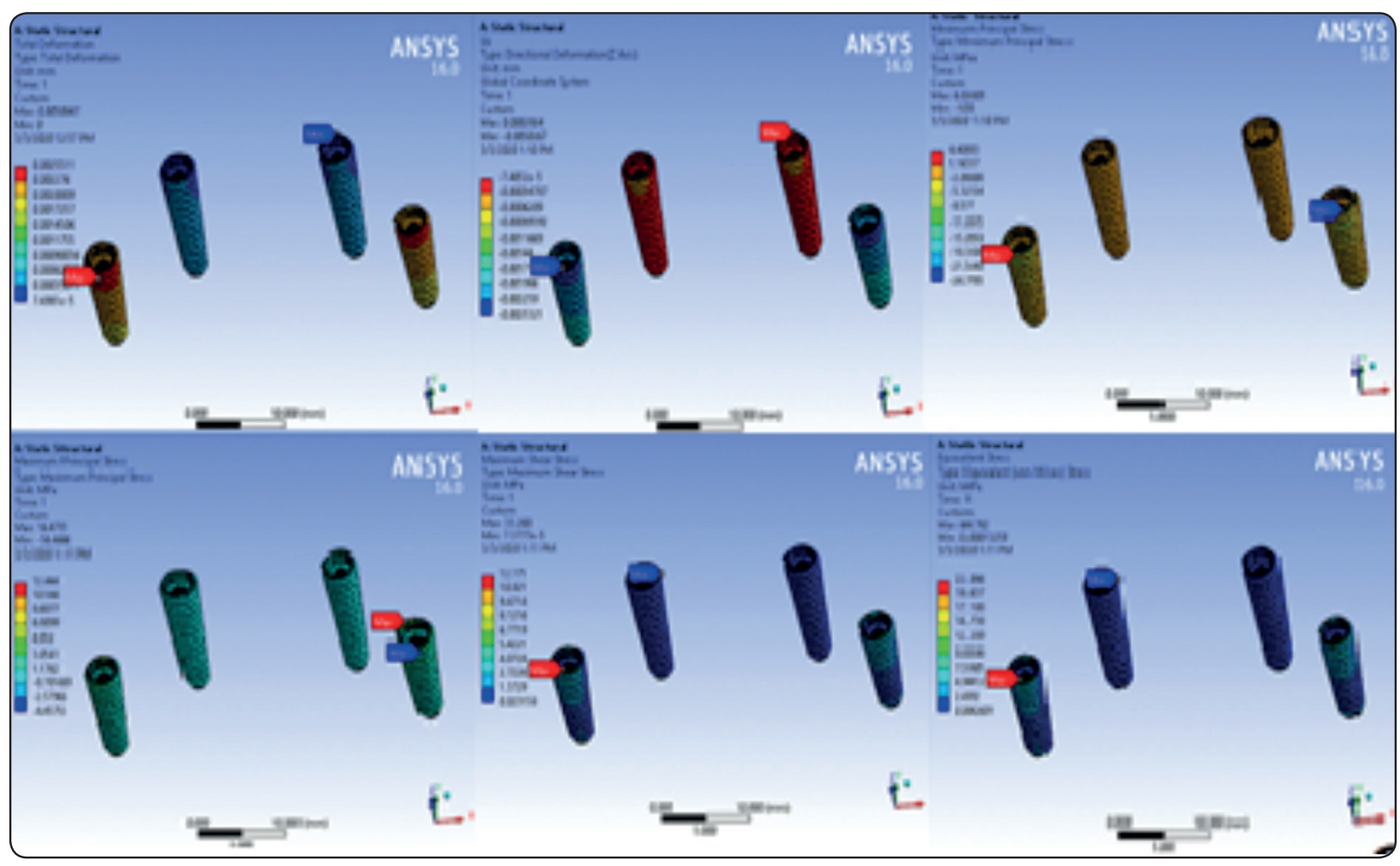

Fig. (1) Different stresses induced within the implants

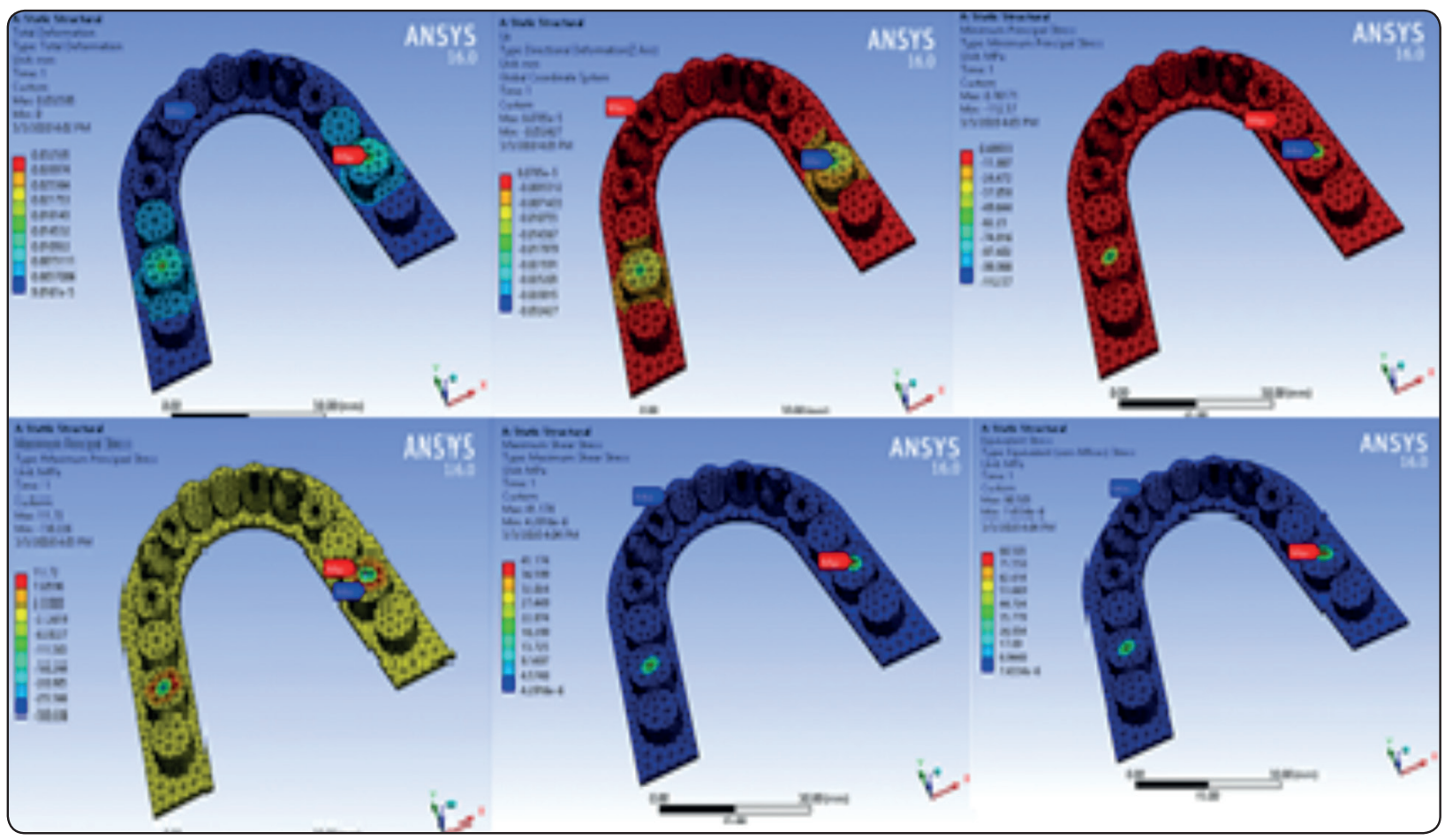

Fig. (2): Different stresses induced within the acrylic restoration (Distal extension) 


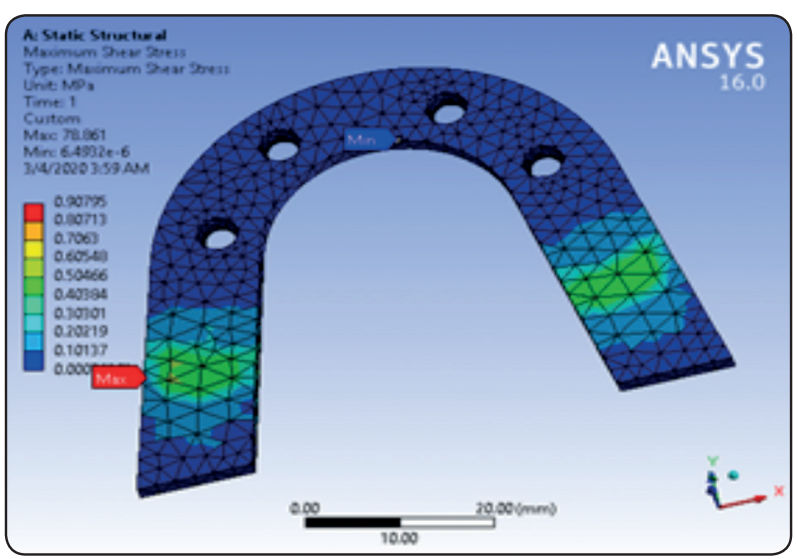

Fig. (3): Stresses induced within the mucosa

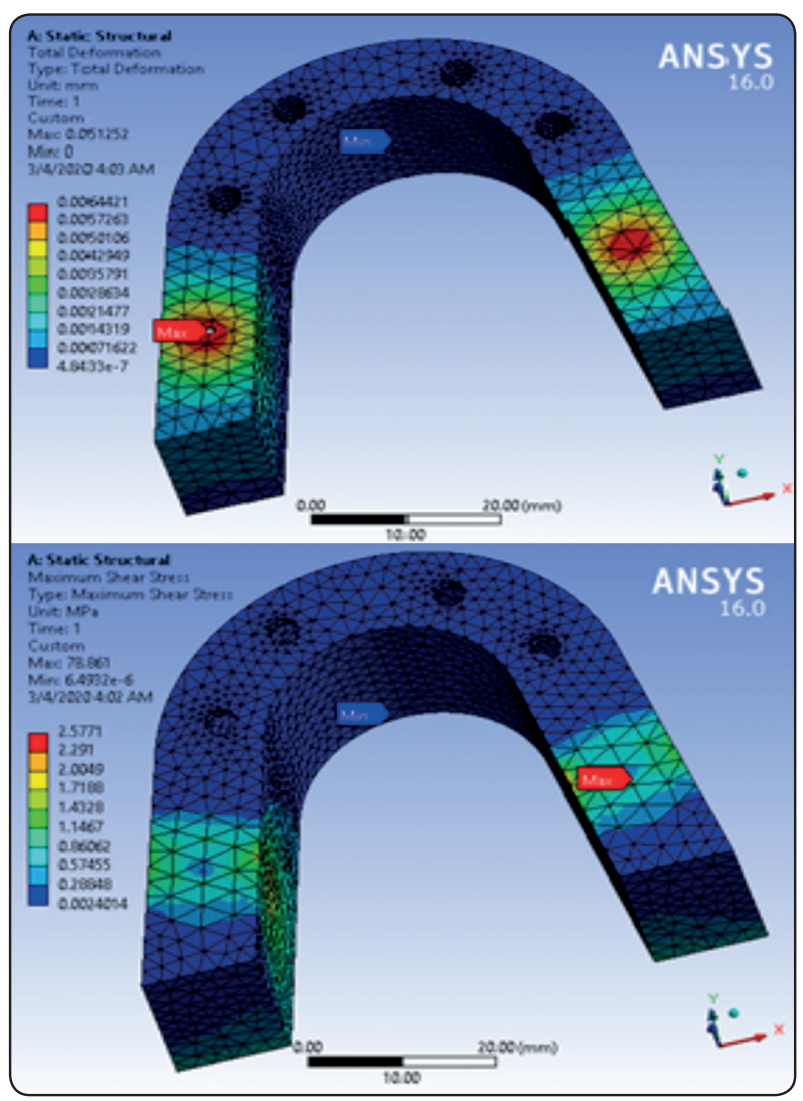

Fig. (4): Stresses induced within the Spongy and Cortical bone

Acrylic screw-retained fixed restorations, Bilateral vertical loading of $100 \mathrm{~N}$ at first molars, $50 \mathrm{~N}$ at second premolars, $16 \mathrm{~mm}$ cantilever length.

In this case, an implant mandibular fixed restoration was tested as the restoration was supported by these implants two at lateral incisors and two at the first pre-molar. The vertically applied load was $100 \mathrm{~N}$ at first molars and $50 \mathrm{~N}$ second premolars bilaterally, where cantilever length is $16 \mathrm{~mm}$ while the fixed restoration material was "Acrylic resin". The following figures are just listing of the different deformation and stresses distributions for each component of the model.

Acrylic screw-retained fixed restorations, Bilateral vertical loading of $100 \mathrm{~N}$ at first molars, $50 \mathrm{~N}$ at second premolars, $20 \mathrm{~mm}$ cantilever length.

In this case, an implant mandibular fixed restoration was tested as the restoration was supported by these implants two at lateral incisors and two at the first pre-molar. The vertically applied load was $100 \mathrm{~N}$ at first molars and $50 \mathrm{~N}$ second premolars bilaterally, where cantilever length is $20 \mathrm{~mm}$ while the fixed restoration material was "Acrylic Resin". The following figures are just listing of the different deformation and stresses distributions for each component of the model.

\section{RESULTS}

Millions of colored pictures were obtained from the finite element analysis and presented here, but for convenience total deformation (Usum), and Von Mesis Stress (Svon) was compared and presented. That gave sufficient information about each component behavior of the studied system.

The effect of cantilever length and type of superstructure material was investigated under two distinct loading protocol (immediate loading). For every loading protocol the stresses generated was be represented as follows:

\section{On Implant}

The implant deformation slightly decreases with the increase of cantilever length. Similarly, implants Von Mises stress clearly decreases while increasing the cantilever extension. Total deformation differences may be neglected as it was less than 
one micron. On the other hand, stress differences changed from about 16.3 to $1.3 \mathrm{MPa}$, that represent about 88 to $58 \%$ respectively.

\section{On abutments}

According to comparison, abutment deformation slightly decreased with the increase of cantilever length. Similarly, Von Misses stress obviously decline while increasing the cantilever length, stress differences changed from about 20 to $0.33 \mathrm{MPa}$, that represent about 93 to $24 \%$ respectively. Total deformation differences did not reach one micron whatever the loading site is.

\section{On screws}

Continuous reduction of screws total deformation and Von Misses stress was recorded with moving loads away from last implant. stress differences changed from about 8.33 to $.134 \mathrm{MPa}$, that represent about 90 to $15 \%$ respectively. All values of deformations and stresses were within physiological limits.

\section{On screw retained fixed restoration (Distal Ex- tension)}

The restoration deformation increases with the increase of cantilever length. Similarly, restoration Von Mises stress dramatically increases while increasing the cantilever extension, it is changed from about 5.6 to $74 \mathrm{MPa}$, that represent about 7 to $90 \%$ respectively.

\section{On Mucosa}

Mucosa directly reflect the screw-retained fixed restoration behavior, that according to the mucosal total deformation and Von Mises stress, slightly increases with the increase of cantilever length.

\section{On peri-implant cortical bone}

While comparing longer cantilevers to shorter cantilever, the LV resulted in an increase in the maximum von Misses stress values among all periimplant bone regions. By moving loading site away from last implant, this induced Von Mises stress on cortical bone of about $40 \%$ increase. Similar trend appeared with total deformation that also recorded $40 \%$ increase.

\section{On Spongy bone}

Spongy bone deformed more when the load application moved away from posterior implants, similarly the Von Mises stress difference increased by about $33 \%$.

All illustrated in figure (5).

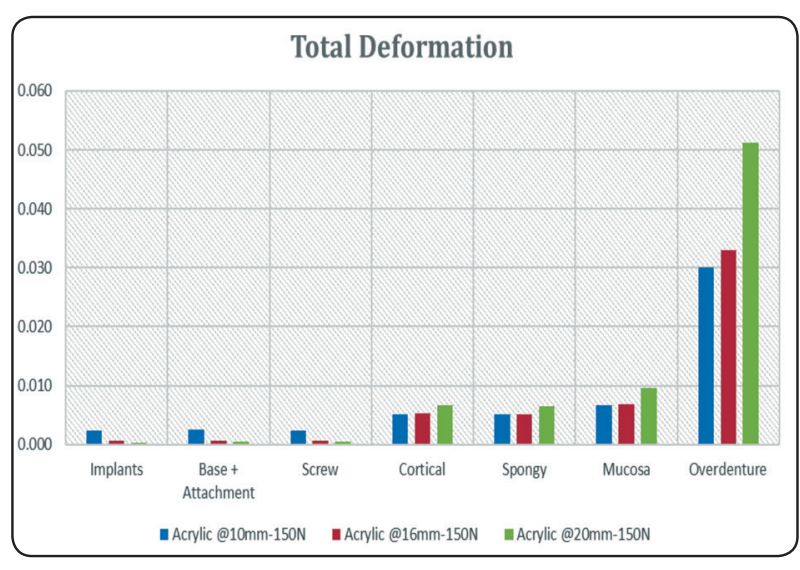

Fig. (5): Bar chart revealing total deformation regarding implants, base attachment, screw, cortical, spongy bone, mucosa, overdenture

\section{DISCUSSION}

This study reveals that on moving the site of load application away from the posterior implant, total deformation and Von Misses stresses of implants, screws and multi-unit abutments decreased, while the stresses on the peri-implant bone and mucosa increased, the screw-retained fixed restoration recorded high values of stresses with increasing the cantilever length.

Previous studies mentioned that stresses were concentrated on the loaded side particularly around the posterior implant. The intensity of the peri-implant stresses faded as the load propagates within the restoration. The degree of stress fading is 
related to the elastic modulus of the superstructure. The lower the elastic modulus, the greater the force applied to the abutment/implant closest to the load temporary acrylic restoration used in this study had relatively low elastic modulus favoring the stress concentration at the posterior loaded side ?

As the material gets stiffer (higher modulus of elasticity), its capacity to absorb the energy resultant from the applied load becomes more; thus transferring less energy to the neighboring structures ${ }^{10}$. The modulus of elasticity of the healing bone used in the immediate loading simulation is almost $50 \%$ lower than cancellous bone and $95 \%$ lower than compact bone; thus it showed low resistance to stress propagation permitting relatively high stress to reach the implant apex. This observation is consistent with the finding of Huang et al who evaluated the effect of implant design on the bone stresses in immediately loaded implants using 3D FEA. ${ }^{11}$

In this study, while comparing longer cantilevers to shorter cantilever, the LV resulted in an increase in the maximum von Misses stress values among all peri-implant bone regions. All values of deformations and stresses were within physiological limits.

The peak stress values recorded with long cantilevers were always higher when compared with short cantilevers. Cantilever arms are force magnifiers which modifed the applied loads into bending moments. The resultant moment is a multiplication of the applied force with the length of the magnification arm. Consequently, long cantilevers would generate higher bending moments on the restorations which in turn transfer more stresses to the peri-implant bone. This finding is consistent with other in vitro studies on cantilever arms. ${ }^{28}$

In a study of the effect of cantilever length on the stress distribution in peri-implant area of cantilevered implant-supported fixed restoration, it was revealed that the center of stress advancement affects the strain on the surrounding structures, advancing with the extension of the lever arm. ${ }^{29}$
Fazi et al., 2011 30 analyzed the stresses at the implant-bone interface; at the external cortical bone surface, radial to the terminal implant; and in the cancellous bone along the implant body, three different meshes with elements of $0.25 \mathrm{~mm}, 0.5 \mathrm{~mm}$, and $1 \mathrm{~mm}$ were used to analyze the bone/implant interface. Most of the stresses transmitted to bony structures are absorbed by cortical bone. Maximum stresses in cancellous bone were found at the disto lingual aspect of the terminal implant on the loaded side and varied from 7.6 to $12.7 \mathrm{MPa}$.

Meanwhile; Mucosa directly reflected the screw-retained fixed restoration behavior, this study reveals that the mucosal total deformation and Von Mises stresses, slightly increases with the increase of cantilever length.

The mucosal total deformation and Von Mises stress under acrylic overdenture showed more value in comparison to PFM one. All values of deformations and stresses were within physiological limits.

It was stated in a study of the effect of cantilever length on the stress distribution in peri-implant area of cantilevered implant-supported fixed restoration, it was reported an increase in the mucosal deformation and stresses with the increase of the lever arm. ${ }^{13}$

It may be attributed to that when the site of load application moves away from the posterior implant, the resultant bending moments is a multiplication of the applied force with the length of the magnification arm. Consequently, long cantilevers would generate higher bending moments on the restorations which in turn transfer more stresses to the surrounding mucosal tissue. This finding is consistent with other in vitro studies on cantilever arms ${ }^{12}$.

This study reveals that the implant deformation slightly decreases with the increase of cantilever length. Similarly, implants Von Mises stress clearly decreases while increasing the cantilever extension.

In coincidence with this study finding, Durkan, Rukiye et al., in a study of influences of cantilever 
extent on the load distribution of lower restorations revealed that higher cantilever length resulted in lower values in posterior implants, they reported that short cantilevers may be preferable because they result in a more favorable distribution of stress than long cantilevers.$^{15}$

El-Anwar and Aboelfadl stated that the number and position of implants supporting the prosthesis together with the masticatory forces, inter-arch distance, and occlusion scheme affect the stress and strain distribution on the implants involved, surrounding bone, and the superstructure complex. Increased forces on the implant unfavorably stimulate bone reduction in the surrounding area causing fibro-integration, which is possibly followed by implant loss ${ }^{16}$.

Holst et $\mathrm{al}^{16}$, in an in vitro study, measured the effect of provisional restoration type on the vertical displacement of implants in a cantilevered full arch prosthesis supported by 4 implants. They compared an acrylic resin with a metal reinforced resin restoration with an $8 \mathrm{~mm}$ posterior cantilever. They concluded that during which stresses were loaded to cantilevers, spread of stresses with metal strengthening appears more encouraging than with non strengthed acrylic resin".

It was stated that the load application on the cantilever arms produces deformation energy in the system that causes flexion. If a great amount of deformation energy is consumed by the framework on a given point, reduction of the transmitted energy happens, decreasing the stress concentration in other sites. ${ }^{18}$

In this study, it was found that continuous reduction of screws, multi-unit abutment total deformation and Von Misses stress was recorded with moving loads away from last implant.

Similar findings were revealed in V. Varinauskas, S. Diliūnas study, that states from the point of view of screw stressing, the cantilever length should not exceed 3 teeth, as their study revealed higher stresses generated on screws on increasing the cantilever arm.$^{19}$
Jacques et $\mathrm{al}^{20}$ investigated the effect of cantilever length on the stress distribution of mandibularcantilevered implant-supported prostheses using a strain gauge method. They found that the abutment deformation was higher with longer cantilever extensions specially with $20 \mathrm{~mm}$ cantilever which drove the conclusion that excessively long cantilever should be avoided.

When the length of the cantilever is maximum, the outer bending component becomes less important, and the vertical bending component becomes more important. Because the distance between implants is increased, the difference between vertical bending moments in the systems 4-1-1-4 and 4-2-2-4 is not significant. On the other hand, it is possible that the overdenture system is more "mobile" with different abutment distribution and cantilever length. Therefore, the screws of other implants absorb the load. ${ }^{(21)}$

\section{CONCLUSION}

Within the limitations of this study, which is finite method is approximate in addition the analysis is not performed on a real structure, but on a model of it. All the results (such as stresses, strains, or displacements) are approximated and the user cannot precisely estimate the difference between the obtained results and the real ones, the following conclusions can be drawn:

1- All values of deformations and stresses appeared on all models components (cortical, spongy bone, implant, attachment, screws, mucosa, and overdenture) were within physiological limits under all cases of load application.

2- Cortical and spongy bone received less stresses and deformation under more rigid restoration that it distributes the applied load in a good manner than lower rigidity one.

3- Increased cantilever length resulted in increased deformation of acrylic screw retained prosthesis except for the implants which had less deformation. 


\section{REFERENCE}

1. Amaral C. F., Gomes R. S., Garcia R. C. M.and DelBel-Gury A. A. Stress distribution of single-implant retained overdenture reinforced with a framework: A finite element analysis study. Journal of Prosthetic Dentistry. 2018;19(5):791-796.

2. Kutkut A., Bertoli E., Frazer R., Pinto-Sinai G., FuentealbaHidalgo R.and Studts J. A systematic review of studies comparing conventional complete denture and implant retained overdenture. Journal of Prosthodontics Research. 2018;62(1):1-9.

3. Ravidà A., Barootchi S., Askar H., Suárez-López del Amo F., Tavelli L.and Wang H. L. Long-term effectiveness of extra-short $(<6 \mathrm{~mm})$ dental implants: A systematic review. International Journal of Oral \& Maxillofacial Implants. 2019;34(1):68-84.

4. Hyland R, Ellis J, Thomason M, El-Feky A, Moynihan P. A qualitative study on patient perspectives of how conventional and implant-supported dentures affect eating. J Dent 2009;37(9):718-23.

5. Rangert B, Krogh PH, Langer B, Van Roekel N. Bending overload and implant fracture: a retrospective clinical analysis. Int J Oral Maxillofac Implants 1995;10(3):32634.

6. Akca K: Finite element stress analysis of the effect of short implant usage in place of cantilever extensions in mandibular posterior edentulism. J Oral Rehabil 2002;29(4):350-6.

7. Seham E. Mohamed; a finite element analysis of stress distribution in all on four system using different framework materials in the mandible, EDJ 2020, Vol 66, 2423:2430.

8. Rubo JH, Capello Souza EA. Finite-element analysis of stress on dental implant prosthesis. Clin Implant Dent Relat Res 2010;12(2):105-13.

9. Wang TM, Leu LJ, Wang J , Lin LD. Effects of prosthesis materials and prosthesis splinting on peri-implant bone stress around implants in poor-quality bone: a numeric analysis. Int J Oral Maxillofac Implants 2002; 17(2):23 1-7.

10. Lihong He; Mechanical behaviour of human enamel and the relationship to its structural and compositional characteristics (Doctor of Philosophy Thesis): (Sydney), Biomaterials Science Research Unit, Faculty of Dentistry, the University of Sydney, May 2008.

11. HUANG, Heng-Li, HSU, Jui-Ting, FUH, Lih-Jyh, et al. Bone stress and interfacial sliding analysis of implant designs on an immediately loaded maxillary implant: a non-linear finite element study. Jthisnal of dentistry, 2008, vol. 36, no 6, p. 409-417.

12. BIDEZ, Martha Warren et MISCH, Carl E. Clinical biomechanics in implant dentistry. Dental Implant Prosthetics, ed, 2005, vol. 2, p. 95-106.

13. Chen X., Mao B., Zhu Z., Yu J., Lu Y., Zhang Q., et al. A three-dimensional finite element analysis of mechanical function for 4 removable partial denture designs with 3 framework materials: CoCr, Ti-6Al-4V alloy and PEEK. Scientific Reports. 2019;9(1):1-10.

14. Fazi, G \& Tellini, S \& Vangi, D \& Branchi, R. ThreeDimensional Finite Element Analysis of Different Implant Configurations for a Mandibular Fixed Prosthesis. The International journal of oral \& maxillofacial implants.2011, 26. $752-9$.

15. Durkan, R., Oyar, P., \& Deste, G. Effects of Cantilever Length and Implant Inclination on the Stress Distribution of Mandibular Prosthetic Restorations Constructed from Monolithic Zirconia Ceramic.2020, International Jthisnal of Oral \& Maxillofacial Imp.

16. El-Anwar MI, Aboelfadl AK. Comparing different bar materials for mandibular implant-supported overdenture: Finite-element analysis. Indian J Dent Res [serial online] 2019 [cited 2020 Jun 7]; 30:716-21. Available from: http:// www.ijdr.in/text.asp, 2019/30.

17. Holst S, Geiselhoeringer H, Wichmann M, Holst A1. The effect of provisional restoration type on micromovement of implants. J Prosthet Dent 2008; 100(3): 173-82.

18. El-Qoubaa Z.and Othman R. Tensile Behavior of Polyetheretherketone Over a Wide Range of Strain Rates. International Journal of Polymer Science. 2015;2015:1-9.

19. V. Varinauskas, S. Diliūnas, M. Kubilius, R. Kubilius ISSN 1392 - 1207. MECHANIKA. 2013 Volume 19(3): 260-263.

20. Jacques LB, Mthisa MS, Suedam V, Souza EA, Rubo $1 \mathrm{H}$. Effect of cantilever length and framework alloy on the stress distribution of mandibular-camilevered implant-supported prostheses. Clin Oral Implants Res 2009;20(7):737-41.

21. Varinauskas, V., Diliūnas, S., Kubilius, M., \& Kubilius, R. Influence of cantilever length on stress distribution in fixation screws of All- Influence of cantilever length on stress distribution in fixation screws of. January 2016, 1-5. https://doi.org/10.5755/j01.mech.19.3.3614 